patients previously infected with another serotype. Preexisting nonneutralizing heterotypic antibodies bind to DENV and facilitate infection of monocytes, which triggers an inflammatory cascade that is eventually responsible for enhanced disease severity. ${ }^{1}$ Recently, Katzelnick et al. found that patients with anti-DENV titers of 1:21 to 1:80 were at the highest risk for severe dengue. ${ }^{2}$ The importance of antibody concentration is particularly relevant, because postvaccination anti-DENV titers wane with time in patients without natural reexposure. ${ }^{3,4}$

Consequently, children who were seronegative before vaccination and whose postvaccination antibody titers wane are most at risk for severe disease. Studies should therefore evaluate whether booster doses could protect these patients from subsequent severe disease.
Klara M. Posfay-Barbe, M.D.

Arnaud G. L'Huillier, M.D.

Geneva University Hospitals

Geneva, Switzerland

arnaud.Ihuillier@hcuge.ch

No potential conflict of interest relevant to this letter was reported.

1. Kurane I, Rothman AL, Livingston PG, et al. Immunopathologic mechanisms of dengue hemorrhagic fever and dengue shock syndrome. Arch Virol Suppl 1994;9:59-64.

2. Katzelnick LC, Gresh L, Halloran ME, et al. Antibody-dependent enhancement of severe dengue disease in humans. Science 2017;358:929-32.

3. Velumani S, Toh YX, Balasingam S, et al. Low antibody titers 5 years after vaccination with the CYD-TDV dengue vaccine in both pre-immune and naïve vaccinees. Hum Vaccin Immunother 2016;12:1265-73

4. Capeding MR, Laot TM, Boaz M, Wartel TA, Crevat D. Immunogenicity and safety of a tetravalent dengue vaccine during a five-year follow-up period. Trials Vaccinol 2015;4:19-23.

DOI: 10.1056/NEJMc1811986

\title{
H4:IC31 Vaccine or BCG Revaccination for Tuberculosis
}

TO THE EDITOR: Nemes and colleagues (July 12 issue) ${ }^{1}$ examined the effects of bacille CalmetteGuérin (BCG) revaccination on reducing the rate of sustained QuantiFERON-TB Gold In-tube assay (QFT) conversion among adolescents in a highrisk setting. One aspect that received relatively little attention in the trial regards the immunologic mechanisms responsible for these effects, which were assessed only by measurement of interferon- $\gamma$ and interleukin-2 T-cell responses. Studies have shown a combination of improved long-term innate or trained immunity (through epigenetic reprogramming of myeloid cells) and adaptive responses after BCG vaccination, which leads to more effective control of mycobacterial and unrelated infections..$^{2-4}$ These mechanisms can also explain the decrease in unrelated respiratory tract infections after BCG revaccination that was observed by the authors. This decrease is consistent with the nonspecific beneficial effects after BCG vaccination that have been observed repeatedly in persons included in epidemiologic studies, ${ }^{5}$ especially in infants, and warrants sustained attention in future investigations.

\author{
Charlotte de Bree, M.D. \\ Reinout van Crevel, M.D., Ph.D. \\ Mihai G. Netea, M.D., Ph.D. \\ Radboud University Medical Center \\ Nijmegen, the Netherlands \\ charlotte.debree@radboudumc.nl
}

No potential conflict of interest relevant to this letter was reported.

1. Nemes E, Geldenhuys H, Rozot V, et al. Prevention of M. tuberculosis infection with H4:IC31 vaccine or BCG revaccination. N Engl J Med 2018;379:138-49.

2. Kleinnijenhuis J, Quintin J, Preijers F, et al. Bacille CalmetteGuerin induces NOD2-dependent nonspecific protection from reinfection via epigenetic reprogramming of monocytes. Proc Natl Acad Sci U S A 2012;109:17537-42.

3. Joosten SA, van Meijgaarden KE, Arend SM, et al. Mycobacterial growth inhibition is associated with trained innate immunity. J Clin Invest 2018;128:1837-51.

4. Goodridge HS, Ahmed SS, Curtis N, et al. Harnessing the beneficial heterologous effects of vaccination. Nat Rev Immunol 2016;16:392-400.

5. Benn CS, Netea MG, Selin LK, Aaby P. A small jab - a big effect: nonspecific immunomodulation by vaccines. Trends Immunol 2013;34:431-9.

DOI: 10.1056/NEJMc1811046 